\title{
AN ALGORITHM FOR COMBINED CODE AND CARRIER PHASE BASED GPS POSITIONING*
}

\author{
XIAO-WEN CHANG and CHRISTOPHER C. PAIGE ${ }^{\dagger}$
}

\author{
School of Computer Science, McGill University, Montreal, Quebec, \\ CanadaH3A2A7. email: chang@cs.mcgill.ca, paige@cs.mcgill.ca
}

\begin{abstract}
.
The Global Positioning System (GPS) is a satellite based navigation system. GPS satellites transmit signals that allow one to quite accurately estimate the location of GPS receivers. In GPS a typical technique for kinematic position estimation is differential positioning where two receivers are used, one receiver is stationary and its exact position is known, the other is roving and its position is to be estimated. We describe the physical situation and give the mathematical model based on the difference of the measurements at the stationary and roving receivers. The model we consider combines both the code and carrier phase measurements. We then present a recursive least squares approach for position estimation. We take full account of the structure of the problem to make our algorithm efficient, and use orthogonal transformations to ensure numerical reliability of the algorithm. Real data test results suggest our algorithm is effective. An additional benefit of this approach is that the drawbacks of double differencing are avoided. The paper could also serve as a straightforward introduction for numerical analysts to an interesting area of GPS.
\end{abstract}

AMS subject classification: 62L $12,65 \mathrm{~F} 20,65 \mathrm{~F} 25$

Key words: Global Positioning System, recursive least squares, orthogonal transformations

\section{Overview of GPS and Geometry of the Problem}

First we give a brief overview for those numerical analysts who are not familiar with the Global Positioning System (GPS). GPS is a satellite based positioning and navigation system. Its basic constellation consists of 24 satellites, all with very accurate clocks, and each continuously transmitting radio waves at fixed frequencies, which a receiver can use to determine its own position. The satellites are moving with respect to the earth, but their positions at any given time can be obtained quite accurately by the receiver, using information transmitted by the satellites. A GPS receiver usually has a much less accurate clock, but can use the measured time a radio signal from a satellite takes to reach it to estimate the satellite to receiver distance. Combining distances from several satellites with

\footnotetext{
* Received October 2002, revised March 2003

${ }^{\dagger}$ This research was supported by NSERC of Canada Grant RGPIN217191-99, FCAR of Quebec Grant 2001-NC-66487, and NSERC-GEOIDE Network Project ENV\#14 for XiaoWen Chang, and by NSERC of Canada Grant RGPIN9236-01 for Chris Paige.
} 


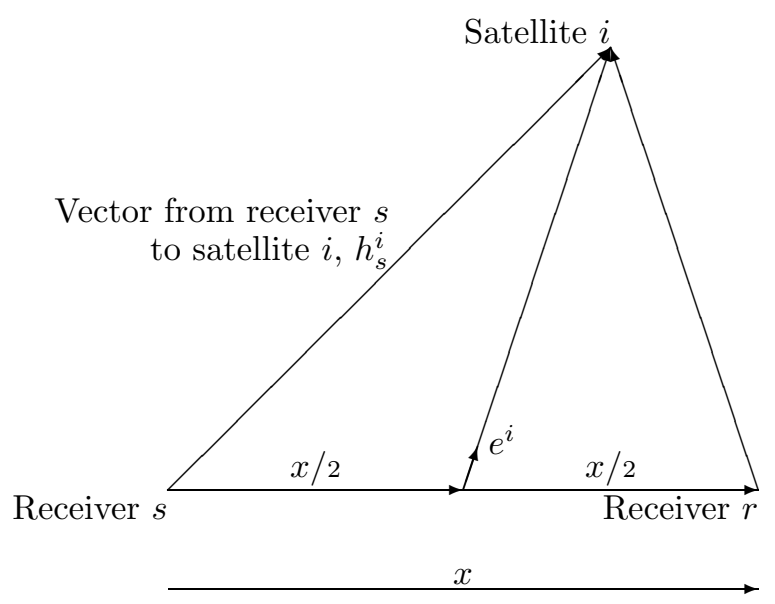

Figure 1.1: Geometry for two Receivers and one Satellite.

satellite positions can lead to an estimate of the receiver's position. In principle at least three visible satellites are needed to estimate the three coordinates of the receiver's position. But because the receiver's clock error can be relatively large (we are timing signals traveling at about $3 \times 10^{10} \mathrm{~cm} / \mathrm{sec}$ ), it has to be treated as an unknown. Thus at least four satellites are required. We would like more in order to obtain a better least squares (LS) estimate of the receiver's position. Usually 6 to 8 satellites are visible from an open area on the earth's surface.

Here we are interested in kinematic position estimation (this term implies a moving object, such as a car or an aircraft). A typical technique for obtaining highly accurate position estimates is relative positioning, see for example [11, $\S 4.8]$. In this case two receivers $s$ and $r$ are used (see Figure 1.1): $s$ is stationary and is set up at a surveyed site whose exact position is known, while $r$ is roving and its position is to be estimated. The purpose of using two receivers is to eliminate some common errors in the GPS signal measurements obtained by the two receivers. We want to find the baseline vector $x \in \mathcal{R}^{3}$, i.e. the vector pointing from receiver $s$ to receiver $r$. If this is known, the position of the roving receiver will be known. In real-time applications there is a radio link between the two receivers so that the information about the stationary receiver and the signal measurements it receives can be sent to the roving receiver.

We need the following quantities to describe the geometry at a given time, that is, the relative positions of satellites and receivers (see Figure 1.1):

$e^{i} \quad$ the unit vector from the midpoint of the baseline to satellite $i$,

$\rho_{s}^{i} \quad$ the range in wavelengths from receiver $s$ to satellite $i$,

$\lambda$ the wavelength (for the L1 carrier signal used here, $\lambda \approx 19 \mathrm{~cm}$ ),

$h_{s}^{i} \quad$ the vector from receiver $s$ to satellite $i$, so $\left\|h_{s}^{i}\right\|=\lambda \rho_{s}^{i}$. 
Note that $\|\cdot\| \equiv\|\cdot\|_{2}$, superscripts indicate satellites, subscripts receivers.

Since $x=h_{s}^{i}-h_{r}^{i}$ and $\left\|2 h_{s}^{i}-x\right\| e^{i}=2 h_{s}^{i}-x=h_{s}^{i}+h_{r}^{i}$, we have

$$
\left(\left\|2 h_{s}^{i}-x\right\| e^{i}\right)^{T} x=\left\|h_{s}^{i}\right\|^{2}-\left\|h_{r}^{i}\right\|^{2}=\left(\left\|h_{s}^{i}\right\|-\left\|h_{r}^{i}\right\|\right)\left(\left\|h_{s}^{i}\right\|+\left\|h_{r}^{i}\right\|\right)
$$

giving

$$
\begin{gathered}
\left(\omega^{i} e^{i}\right)^{T} x=\lambda\left(\rho_{s}^{i}-\rho_{r}^{i}\right), \\
\omega^{i} \equiv \frac{\left\|2 h_{s}^{i}-x\right\|}{\left\|h_{s}^{i}\right\|+\left\|h_{s}^{i}-x\right\|}, \quad e^{i}=\frac{2 h_{s}^{i}-x}{\left\|2 h_{s}^{i}-x\right\|} .
\end{gathered}
$$

We will have available a close approximation to $h_{s}^{i}$. The true baseline vector $x$ will not be known, but we will see that at each step we will have an estimate of it, and this will be used to evaluate $\omega^{i} e^{i}$ above.

The stationary and roving receivers obtain measurements of $\rho_{s}^{i}$ and $\rho_{r}^{i}$ respectively. Thus if there were no errors, measurements from $m$ satellites would give $m$ equations of the form (1.1). Just as in the case where one receiver is used for positioning, at least $m \geq 4$ visible satellites are required here to estimate $x$, since the difference of the two receivers' clock errors has to be treated as an unknown.

The signals which give $\rho_{s}^{i}$ and $\rho_{r}^{i}$ above are called code signals. Unfortunately these only give mediocre position accuracy (to at best a few metres accuracy). Receivers can also use what are called carrier signals. These give much greater accuracy, but are more complicated to use, in that they do not supply $\rho_{s}^{i}$ or $\rho_{r}^{i}$ directly. Instead, at time $t$, they supply what is called the carrier phase measurement, for example $\phi_{s}^{i}(t)$, where in the absence of errors

$$
\rho_{s}^{i}(t)=\phi_{s}^{i}(t)+\alpha_{s}^{i}
$$

Here $\alpha_{s}^{i}$ is an unknown, time independent quantity called the ambiguity. Thus at a given time $t$, instead of having $m$ equations of the form (1.1) for $x(t)$, in the absence of errors the carrier signals would give $m$ equations of the form

$$
\left[\lambda^{-1} \omega^{i}(t) e^{i}(t)^{T},-1\right]\left[\begin{array}{c}
x(t) \\
\alpha^{i}
\end{array}\right]=\phi_{s}^{i}(t)-\phi_{r}^{i}(t),
$$

where $\alpha^{i} \equiv \alpha_{s}^{i}-\alpha_{r}^{i}$ is a new unknown for each satellite. In reality we also have to account for noise and inaccurate clocks, just as we do for code signals. Even with $m \geq 5$ visible satellites, measurements are required at several time steps $t_{k}$ before estimates of the $\alpha^{i}$ and $x\left(t_{k}\right)$ can be obtained, and it can take many time steps to obtain estimates with small errors. This suggests that it would be useful to find estimates using both the code and the carrier phase measurements - the code measurements should help to give good answers quickly, while the carrier phase measurements should help to give greater accuracy.

As well as clock errors there are measurement noises, and the signals suffer ionospheric and tropospheric refraction errors, among others. We assume the baseline is short (less than $20 \mathrm{~km}$ say) so that a satellite signal has almost the 
same ionospheric refraction error, and the same tropospheric refraction error, at the two receivers. We almost always difference the two signal measurements from the same satellite at the two receivers, see $\rho_{s}^{i}-\rho_{r}^{i}$ in (1.1), to essentially eliminate these two refraction errors. This also eliminates the satellite clock error. This technique is called single differencing (see [6]).

One can choose a particular satellite to be the "reference satellite", then difference the single differenced measurements from the reference satellite with those from the other satellites. This is called double differencing (see [2]). Double differencing can eliminate the two receivers' clock errors. Double differencing is widely used in GPS computations, but it has some drawbacks. For example it is numerically slightly dubious, it makes the double differenced measurements correlated, and it gives unnecessary prominence to the reference satellite. In [4] we presented an orthogonal transformation approach for carrier phase measurements which avoided the first two drawbacks, while maintaining all the advantages of double differencing.

The approach presented in [4] is numerically reliable and efficient. The main purpose of this paper is to extend that approach to the combined case where both carrier phase and code measurements are used for positioning. This leads to the mathematical model (3.8) corresponding to time step $t_{k}$. There $x_{k}$ is the baseline, $\beta_{k}$ is the difference of the two receivers' clock errors, and $a$ is the vector of ambiguity differences $\alpha^{i}$. In Section 4 we design a recursive QR factorization to obtain the LS estimates of the $x_{k}$ for a sequence of such time steps. In [4] we avoided double differencing the measurements, but we still had to involve double differenced ambiguities or their equivalents. Since more information is available here, we are able to avoid some problems like the reference satellite issue, which previously complicated the handling of rising and setting satellites.

This paper has been written to be easily accessible for any reader with a basic knowledge of numerical linear algebra, and is organized as follows. We continue to use the geometry of Figure 1.1, but will now pay careful attention to the errors that occur in the measurements used to replace $\rho_{s}^{i}$ and $\rho_{r}^{i}$ in (1.1). In Section 2 we give some GPS background. In Section 3 we derive the mathematical model we use for position estimation. In Section 4 we show how to use orthogonal transformations to make full use of the structure of the model to compute the LS estimates of the positions. In Section 5 we give the results of tests with real data. A summary and some remarks are given in Section 6 .

We will use $v \sim \mathcal{N}(\bar{v}, V)$ to indicate that $v$ is a normally distributed random vector with expected value $\bar{v}$ and covariance $V$.

\section{Some GPS details}

In this section we give some GPS details needed to put our approach in context. The reader can safely skip this section on the first reading. Each GPS satellite continuously transmits two carrier radio waves L1 and L2 with precise frequencies $1575.42 \mathrm{MHz}$ and 1227.60 MHZ, respectively. Superimposed on the L1 carrier are the $\mathrm{C} / \mathrm{A}$ code (coarse acquisition) and the P-code (precision), while superimposed on the L2 carrier is only the P-code. The C/A code is for 
civilian users, while the P-code is for US military or authorized users.

Currently there are hundreds of GPS receiver models on the market for different applications. GPS receivers for commercial aircraft may cost more than US $\$ 10,000$; but easily available pocket-sized receivers may cost less than US $\$ 100$. Many receivers are designed to receive only the L1 carrier mainly for economical reasons. As in [4], we only consider the L1 carrier, but it is easy to extend our approach to the dual frequency case. Basically there are two kinds of measurements which can be used for positioning: code (also called pseudorange, or code pseudorange; we will consider the C/A code) and carrier phase. Both measurements are subject to ionospheric refraction errors, tropospheric refraction errors, the receiver clock error, the satellite clock error, multipath errors (caused by reflections), and random measurement noise, see for example [11, §4.1] for more details. As we mentioned earlier, the code measurement gives an approximation to the true range between the receiver and the satellite, and can easily be used for positioning. Using the carrier phase measurement for position estimation is not nearly so easy, but it is much more accurate than the code measurement.

In [4] only carrier phase data was used for positioning. But it is now common to use both carrier phase and code data to obtain better results. In fact much research has been conducted on integrating these two types of measurement. For example Hatch [7], Ashjaee [1] and Goad [5] studied how to generate the so-called carrier-smoothed code measurements by using carrier phase and code measurements. Kleusberg [10], Hwang and Brown [8], Teunissen [12], Jin [9] and Tiberius [13] investigated how to use carrier phase and code measurements in GPS kinematic positioning. Most methods assume that a dynamic model for the roving receiver is available, and then use the standard Kalman filter. The main differences among these methods are that different information is assumed to be sent from the stationary receiver to the roving receiver, and different dynamic models are used. Most methods given in the GPS literature do not address the computer implementation issue. Even when they do, they usually do not take full advantage of the structure of the model. In this paper we assume the carrier phase and code measurements of the stationary receiver are available at the roving receiver. We do not use any dynamic model, because for many practical applications it is hard to model the dynamic behaviour of the roving receiver. GPS measurements are extremely accurate, and we believe that artificially including a dynamic model may degrade the estimation performance, while the additional computation in each epoch is unnecessarily expensive. However when a sufficiently accurate dynamic model is known, it can be included in the full model here, and we believe the approach given here can be extended to this.

\section{The mathematical model}

Here we give the mathematical model we use for position estimation, however we omit some minor subtleties in order to simplify the presentation.

Suppose the signal from satellite $i$ arrives at receiver $s$ at epoch $k$ (that is, at time point $\left.t_{k}\right)$ and its travel time is $t_{s}^{i}\left(t_{k}\right)$. At $t_{k}$ the carrier phase measurement $\phi_{s}^{i}\left(t_{k}\right)$ (in wavelengths, cf. (1.3)) and the code measurement $\tilde{\rho}_{s}^{i}\left(t_{k}\right)$ (in 
wavelengths) at receiver $s$ for satellite $i$ are (see for example [11, §4.1])

$$
\begin{aligned}
\phi_{s}^{i}\left(t_{k}\right)+\alpha_{s}^{i} & =\rho_{s}^{i}\left(t_{k}\right)-\iota_{s}^{i}\left(t_{k}\right)+\tau_{s}^{i}\left(t_{k}\right)+\beta^{i}\left(t_{k}-t_{s}^{i}\left(t_{k}\right)\right)+\beta_{s}\left(t_{k}\right)+\mu_{s}^{i}\left(t_{k}\right), \\
\tilde{\rho}_{s}^{i}\left(t_{k}\right) & =\rho_{s}^{i}\left(t_{k}\right)+\iota_{s}^{i}\left(t_{k}\right)+\tau_{s}^{i}\left(t_{k}\right)+\beta^{i}\left(t_{k}-t_{s}^{i}\left(t_{k}\right)\right)+\beta_{s}\left(t_{k}\right)+\nu_{s}^{i}\left(t_{k}\right),
\end{aligned}
$$

(the signs preceding the $\iota_{s}^{i}\left(t_{k}\right)$ are correct, see [11, (4.25) \& p.157]) where the "units" of each of the following components is "number of wavelengths":

- $\phi_{s}^{i}\left(t_{k}\right)$ : the carrier phase measurement at time $t_{k}$. Initially $\phi_{s}^{i}\left(t_{1}\right)<1$.

- $\alpha_{s}^{i}$ : the ambiguity. $\phi_{s}^{i}\left(t_{1}\right)+\alpha_{s}^{i}$ is the initial number of wavelengths between satellite $i$ at time $t_{1}-t_{s}^{i}\left(t_{1}\right)$ and receiver $s$ when receiver $s$ starts tracking the satellite signal at $t_{1} . \alpha_{s}^{i}$ is not necessarily an integer. In [13, §3.4.1.1] it includes initial phase offsets of the receiver-generated carrier signal and the satellite-generated signal, which are not taken into account in [11] where $\alpha_{s}^{i}$ is assumed to be an integer. $\alpha_{s}^{i}$ is unknown, but remains the same while tracking is continued without loss of lock.

- $\tilde{\rho}_{s}^{i}\left(t_{k}\right)$ : the code measurement at time $t_{k}$.

- $\rho_{s}^{i}\left(t_{k}\right)$ : the actual range between receiver $s$ at the signal arrival time $t_{k}$ and satellite $i$ at the signal departure time $t_{k}-t_{s}^{i}\left(t_{k}\right)$.

- $\iota_{s}^{i}\left(t_{k}\right)$ : the ionospheric range error at time $t_{k}$.

- $\tau_{s}^{i}\left(t_{k}\right)$ : the tropospheric range error at time $t_{k}$.

- $\beta^{i}\left(t_{k}-t_{s}^{i}\left(t_{k}\right)\right)$ : the satellite clock range error at transmission time $t_{k}-t_{s}^{i}\left(t_{k}\right)$.

- $\beta_{s}\left(t_{k}\right)$ : the receiver clock range error at time $t_{k}$.

- $\mu_{s}^{i}\left(t_{k}\right)$ : the carrier measurement noise, including multipath error, at $t_{k}$.

- $\nu_{s}^{i}\left(t_{k}\right)$ : the code measurement noise, including multipath error, at time $t_{k}$.

The model (3.1)-(3.2) is often used in the literature, although there are more complicated models which take other factors into account, see for example [13]. It might help some readers if we give an intuitive (and approximate) explanation of the model we use. The code measurement is more straightforward. Suppose the satellite sent a code message saying when that message was sent, and the receiver noted the time $t$ it received that message. If the satellite and receiver clocks were synchronized and there were no errors, the receiver would know exactly how long that message took to travel from the satellite to the receiver. Multiplying this time interval by the speed of light in vacuum, and dividing the result by the L1 wavelength would give $\tilde{\rho}_{s}^{i}(t)$, the code measurement at time $t$, that is, the number of wavelengths that would have been between where the satellite was when it sent the message, and where the receiver was when it received the message, if there had been a vacuum between them. Corrections would be added to this to more closely match reality, see (3.2).

The carrier equation (3.1) is more subtle, and the literature on it is often difficult to follow. Here we give an amateurish minor extrapolation of the simplified description in $[11, \S 4.1 .2]$. At a given point in space, the fractional phase difference between two signals of the same frequency $f=1 / T$ is the fraction of 
a wavelength that one is ahead of the other. Thus $\cos (2 \pi t / T)$ is one quarter wavelength ahead of $\sin (2 \pi t / T)$. Essentially the receiver generates a sinusoidal signal to match the frequency of the incoming signal (L1 carrier wave here) from the satellite. It measures the fractional phase difference $\phi_{s}^{i}\left(t_{1}\right)$ between the two signals at the initial time $t_{1}$, and tracks how this phase difference $\phi_{s}^{i}\left(t_{k}\right)$ changes with time. Now consider the error free case in vacuum. Initially the range $\rho_{s}^{i}\left(t_{1}\right)$ (see the definitions above) is just $\phi_{s}^{i}\left(t_{1}\right)+\alpha_{s}^{i}$ for some unknown number of wavelengths $\alpha_{s}^{i}$. If the satellite and receiver stayed in the same positions, each term would stay constant in time. Now suppose the satellite moves one wavelength further away (radially) from the receiver, then the phase difference measurement increases by one wavelength, and in general $\rho_{s}^{i}\left(t_{k}\right)=\phi_{s}^{i}\left(t_{k}\right)+\alpha_{s}^{i}$ for the same unknown number of wavelengths $\alpha_{s}^{i}$. Corrections are added to this to more closely match reality, see (3.1).

Subtracting the equation corresponding to receiver $r$ from (3.1) and noticing $-\left[\iota_{s}^{i}\left(t_{k}\right)-\iota_{r}^{i}\left(t_{k}\right)\right]+\left[\tau_{s}^{i}\left(t_{k}\right)-\tau_{r}^{i}\left(t_{k}\right)\right]+\left[\beta^{i}\left(t_{k}-t_{s}^{i}\left(t_{k}\right)\right)-\beta^{i}\left(t_{k}-t_{r}^{i}\left(t_{k}\right)\right)\right]$ will be negligible since the baseline is short, we obtain the single difference carrier equation

$\phi_{s}^{i}\left(t_{k}\right)-\phi_{r}^{i}\left(t_{k}\right)=\rho_{s}^{i}\left(t_{k}\right)-\rho_{r}^{i}\left(t_{k}\right)-\left(\alpha_{s}^{i}-\alpha_{r}^{i}\right)+\beta_{s}\left(t_{k}\right)-\beta_{r}\left(t_{k}\right)+\mu_{s}^{i}\left(t_{k}\right)-\mu_{r}^{i}\left(t_{k}\right)$.

Receivers $s$ and $r$ occur in every equation, so we can drop these indices and then indicate the time epoch $k$ by subscript $k$ if we define

$\phi_{k}^{i} \equiv \phi_{s}^{i}\left(t_{k}\right)-\phi_{r}^{i}\left(t_{k}\right), \quad \alpha^{i} \equiv \alpha_{s}^{i}-\alpha_{r}^{i}, \quad \beta_{k} \equiv \beta_{s}\left(t_{k}\right)-\beta_{r}\left(t_{k}\right), \quad \mu_{k}^{i} \equiv \mu_{s}^{i}\left(t_{k}\right)-\mu_{r}^{i}\left(t_{k}\right)$.

This with (1.1) gives for (3.3)

$$
\phi_{k}^{i}=\lambda^{-1}\left(\omega_{k}^{i} e_{k}^{i}\right)^{T} x_{k}-\alpha^{i}+\beta_{k}+\mu_{k}^{i} .
$$

By defining $\tilde{\rho}_{k}^{i} \equiv \tilde{\rho}_{s}^{i}\left(t_{k}\right)-\tilde{\rho}_{r}^{i}\left(t_{k}\right)$, we obtain the single difference code measurement equation from $(3.2)$

$$
\tilde{\rho}_{k}^{i}=\lambda^{-1}\left(\omega_{k}^{i} e_{k}^{i}\right)^{T} x_{k}+\beta_{k}+\nu_{k}^{i}
$$

In the usual model, the $\mu_{k}^{i}$ for different satellites and different epochs are assumed to be unbiased independently distributed noises with the same normal distribution. So are the $\nu_{k}^{i}$. Furthermore $\mu_{k}^{i}$ and $\nu_{l}^{j}$ are assumed to be independent. Thus assuming we have $m$ satellites, and writing

$$
\begin{aligned}
y_{k}^{T} \equiv\left[\phi_{k}^{1}, \ldots, \phi_{k}^{m}\right], & z_{k}^{T} \equiv\left[\tilde{\rho}_{k}^{1}, \ldots, \tilde{\rho}_{k}^{m}\right], & E_{k}^{T} \equiv \lambda^{-1}\left[\left(\omega_{k}^{1} e_{k}^{1}\right), \ldots,\left(\omega_{k}^{m} e_{k}^{m}\right)\right] \\
a^{T} \equiv\left[\alpha^{1}, \ldots, \alpha^{m}\right], & u_{k}^{T} \equiv\left[\mu_{k}^{1}, \ldots, \mu_{k}^{m}\right], & v_{k}^{T} \equiv\left[\nu_{k}^{1}, \ldots, \nu_{k}^{m}\right]
\end{aligned}
$$

and using the standard notation $e^{T} \equiv[1, \ldots, 1]$, we have (where typical values of the standard deviations $\sigma_{\phi}$ and $\sigma_{\rho}$ are available from the literature):

$$
\begin{array}{rlrl}
y_{k} & =E_{k} x_{k}-a+e \beta_{k}+u_{k}, & u_{k} & \sim \mathcal{N}\left(0, \sigma_{\phi}^{2} I_{m}\right), \\
z_{k}=E_{k} x_{k}+e \beta_{k}+v_{k}, & v_{k} \sim \mathcal{N}\left(0, \sigma_{\rho}^{2} I_{m}\right) .
\end{array}
$$


These are the desired single differences of measurements equations given the physical situation of $m$ satellites with a known fixed receiver and a roving receiver whose position is to be estimated. Let $\sigma \equiv \sigma_{\phi} / \sigma_{\rho}$. In order to make the noise vector in (3.7) have the same standard deviation as that in (3.6), we multiply (3.7) by $\sigma$, then write the resulting equation and (3.6) together

$$
\left[\begin{array}{c}
y_{k} \\
\sigma z_{k}
\end{array}\right]=\left[\begin{array}{c}
E_{k} \\
\sigma E_{k}
\end{array}\right] x_{k}-\left[\begin{array}{c}
I \\
0
\end{array}\right] a+\left[\begin{array}{c}
I \\
\sigma I
\end{array}\right] e \beta_{k}+\left[\begin{array}{c}
u_{k} \\
\sigma v_{k}
\end{array}\right], \quad\left[\begin{array}{c}
u_{k} \\
\sigma v_{k}
\end{array}\right] \sim \mathcal{N}\left(0, \sigma_{\phi}^{2} I_{2 m}\right)
$$

\section{Efficient and numerically reliable position estimation}

Following [4], we will present a recursive LS algorithm by orthogonal transformations to estimate positions based on the carrier phase and code measurement equations (3.8). Notice that in $E_{k}, \omega_{k}^{i} e_{k}^{i}$ depends on the baseline $x_{k}$, see (1.2) and (3.5). So we may write

$$
E_{k} \equiv E\left(x_{k}\right)
$$

This $E_{k}$ is known once $x_{k}$ is known. Given an approximation to $x_{k}$ (because $E(x)$ is not very sensitive to changes in $x$, our estimate of $x_{k-1}$ is usually sufficient), we can compute our approximation to $E_{k}$. Then given the measurements $y_{k}$ and $z_{k}$, we can obtain a better estimate of $x_{k}$, and of $E_{k}$ if necessary. But to save space here we just assume that the $E_{k}$ in (3.8) are known, and that the number of visible satellites does not change during the observation period.

First we eliminate $x_{k}$ and $\beta_{k}$ from the lower half of (3.8). Define orthogonal

$G \equiv\left[\begin{array}{cc}c I & s I \\ -s I & c I\end{array}\right]$, where $\gamma \equiv \sqrt{1+\sigma^{2}}, c \equiv 1 / \gamma, s \equiv \sigma / \gamma$, so $G\left[\begin{array}{c}I \\ \sigma I\end{array}\right]=\left[\begin{array}{c}\gamma I \\ 0\end{array}\right]$.

Then applying $G$ to (3.8), we obtain

$$
\left[\begin{array}{c}
c y_{k}+s \sigma z_{k} \\
-s y_{k}+c \sigma z_{k}
\end{array}\right]=\left[\begin{array}{c}
\gamma I \\
0
\end{array}\right] E_{k} x_{k}-\left[\begin{array}{c}
c I \\
-s I
\end{array}\right] a+\left[\begin{array}{c}
\gamma I \\
0
\end{array}\right] e \beta_{k}+\left[\begin{array}{c}
c u_{k}+s \sigma v_{k} \\
-s u_{k}+c \sigma v_{k}
\end{array}\right]
$$

Now we eliminate the clock bias $\beta_{k}$. Following [4], let $P \in \mathcal{R}^{m \times m}$ be a Householder transformation such that

$$
P e=e_{1} \sqrt{m}, \quad P \equiv I-u\left(\frac{2}{u^{T} u}\right) u^{T}, \quad u \equiv e_{1}-e / \sqrt{m} .
$$

Partition $P \equiv\left[\begin{array}{c}p^{T} \\ \bar{P}\end{array}\right]$, then multiplying (4.1) by $\operatorname{diag}(P, I)$ from the left, we obtain

$$
\left[\begin{array}{c}
p^{T}\left(c y_{k}+s \sigma z_{k}\right) \\
\bar{P}\left(c y_{k}+s \sigma z_{k}\right) \\
-s y_{k}+c \sigma z_{k}
\end{array}\right]=\left[\begin{array}{c}
\gamma p^{T} E_{k} \\
\gamma \bar{P} E_{k} \\
0
\end{array}\right] x_{k}-\left[\begin{array}{c}
c p^{T} \\
c \bar{P} \\
-s I
\end{array}\right] a+\left[\begin{array}{l}
1 \\
0 \\
0
\end{array}\right] \sqrt{m} \gamma \beta_{k}+\left[\begin{array}{c}
p^{T}\left(c u_{k}+s \sigma v_{k}\right) \\
\bar{P}\left(c u_{k}+s \sigma v_{k}\right) \\
-s u_{k}+c \sigma v_{k}
\end{array}\right]
$$

Notice that only the first equation in (4.3) involves the clock bias term $\beta_{k}$, and we will see later $((4.7)$ and the line preceding it) that this equation can be dropped for position estimation. For estimating $x_{k}$ later, we would like to 
transform $\gamma \bar{P} E_{k}$ to upper triangular form. Let the QR factorization of $\gamma \bar{P} E_{k}$ be (we assume $m \geq 4$ so that $\gamma \bar{P} E_{k}$ almost always has full column rank, see for example $[4, \S 3.2])$

$$
Q_{k}^{T}\left(\gamma \bar{P} E_{k}\right)=\left[\begin{array}{c}
R_{k} \\
0
\end{array}\right], \quad Q_{k}^{T}=\left[\begin{array}{c}
U_{k} \\
V_{k}
\end{array}\right], \quad 3 \times(m-1) U_{k}, \quad(m-4) \times(m-1) V_{k} .
$$

Multiplying (4.3) by $\operatorname{diag}\left(1, Q_{k}^{T}, I_{m}\right)$ from the left gives

$$
\left[\begin{array}{c}
p^{T}\left(c y_{k}+s \sigma z_{k}\right) \\
U_{k} \bar{P}\left(c y_{k}+s \sigma z_{k}\right) \\
V_{k} \bar{P}\left(c y_{k}+s \sigma z_{k}\right) \\
-s y_{k}+c \sigma z_{k}
\end{array}\right]=\left[\begin{array}{c}
\gamma p^{T} E_{k} \\
R_{k} \\
0 \\
0
\end{array}\right] x_{k}-\left[\begin{array}{c}
c p^{T} \\
c U_{k} \bar{P} \\
c V_{k} \bar{P} \\
-s I
\end{array}\right] a+\left[\begin{array}{c}
1 \\
0 \\
0 \\
0
\end{array}\right] \sqrt{m} \gamma \beta_{k}+\left[\begin{array}{c}
p^{T}\left(c u_{k}+s \sigma v_{k}\right) \\
U_{k} \bar{P}\left(c u_{k}+s \sigma v_{k}\right) \\
V_{k} \bar{P}\left(c u_{k}+s \sigma v_{k}\right) \\
-s u_{k}+c \sigma v_{k}
\end{array}\right] .
$$

Denote

$$
\begin{array}{ll}
\eta_{k} \equiv p^{T}\left(c y_{k}+s \sigma z_{k}\right), & \delta_{k} \equiv p^{T}\left(c u_{k}+s \sigma v_{k}\right), \\
\bar{y}_{k} \equiv U_{k} \bar{P}\left(c y_{k}+s \sigma z_{k}\right), & \bar{u}_{k} \equiv U_{k} \bar{P}\left(c u_{k}+s \sigma v_{k}\right), \\
\bar{z}_{k} \equiv V_{k} \bar{P}\left(c y_{k}+s \sigma z_{k}\right), & \bar{v}_{k} \equiv V_{k} \bar{P}\left(c u_{k}+s \sigma v_{k}\right), \\
g_{k} \equiv-s y_{k}+c z_{k}, & f_{k} \equiv-s u_{k}+c \sigma v_{k} .
\end{array}
$$

Then (4.5) can be written as

$$
\left[\begin{array}{c}
\eta_{k} \\
\bar{y}_{k} \\
\bar{z}_{k} \\
g_{k}
\end{array}\right]=\left[\begin{array}{c}
\gamma p^{T} E_{k} \\
R_{k} \\
0 \\
0
\end{array}\right] x_{k}-\left[\begin{array}{c}
c p^{T} \\
c U_{k} \bar{P} \\
c V_{k} \bar{P} \\
-s I
\end{array}\right] a+\left[\begin{array}{l}
1 \\
0 \\
0 \\
0
\end{array}\right] \sqrt{m} \gamma \beta_{k}+\left[\begin{array}{c}
\delta_{k} \\
\bar{u}_{k} \\
\bar{v}_{k} \\
f_{k}
\end{array}\right]
$$

Combining these for $k=1,2, \ldots$ and reordering gives with $\gamma_{k} \equiv \sqrt{m} \gamma \beta_{k}$

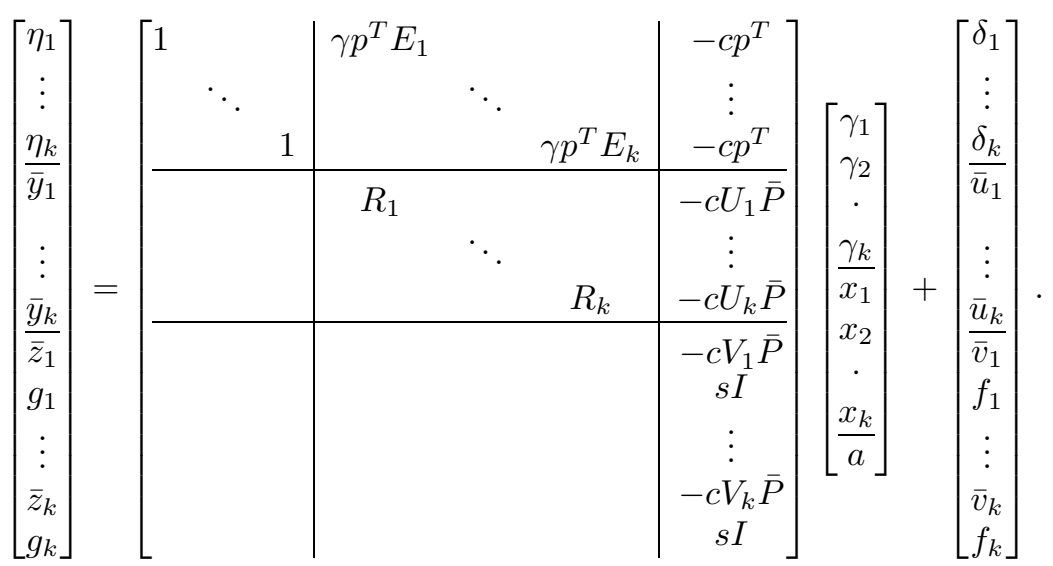

Since orthogonal transformations preserve the normal distribution, the transformed noise vector follows the distribution $\mathcal{N}\left(0, \sigma_{\phi}^{2} I_{2 k m}\right)$. From the structure of the coefficient matrix in (4.7) we see estimates of $\gamma_{1}, \ldots, \gamma_{k}$ (and so of $\beta_{1}, \ldots, \beta_{k}$ ) 
could easily be found from the first $k$ equations of (4.7) once the estimates of $x_{1}, \ldots, x_{k}$ and $a$ are available. But the $\gamma_{k}$ and $\beta_{k}$ are not usually wanted.

In order to obtain LS estimates of the position, we need only find LS estimates for the sub-model formed by the last $k(2 m-1)$ equations of (4.7). Let $a_{k \mid k}$ denote the LS estimate of $a$ at epoch $k$, then we see that $a_{k \mid k}$ is the LS estimate for the even smaller sub-model:

$$
\left[\begin{array}{c}
\bar{z}_{1} \\
g_{1} \\
\vdots \\
\bar{z}_{k} \\
g_{k}
\end{array}\right]=\left[\begin{array}{c}
-c V_{1} \bar{P} \\
s I \\
\vdots \\
-c V_{k} \bar{P} \\
s I
\end{array}\right] a+\left[\begin{array}{c}
\bar{v}_{1} \\
f_{1} \\
\vdots \\
\bar{v}_{k} \\
f_{k}
\end{array}\right]
$$

Once $a_{k \mid k}$ has been computed, we see from (4.7) that $x_{1 \mid k}, x_{2 \mid k}, \ldots, x_{k \mid k}$, the LS estimates of $x_{1}, x_{2}, \ldots, x_{k}$ at epoch $k$, can be computed by solving the upper triangular systems

$$
R_{j} x_{j \mid k}=\bar{y}_{j}+c U_{j} \bar{P} a_{k \mid k}, \quad j=1, \ldots, k .
$$

Now our task is to obtain the estimate $a_{k \mid k}$ of $a$ from (4.8). We use a recursive approach. Suppose at epoch $k-1$ we have computed the following orthogonal transformations (since $s>0, S_{k-1}$ has full column rank):

$$
T_{k-1}^{T}\left[\begin{array}{c}
-c V_{1} \bar{P} \\
s I \\
\cdot \\
-c V_{k-1} \bar{P} \\
s I
\end{array}\right]=\left[\frac{S_{k-1}}{0}\right], \quad T_{k-1}^{T}\left[\begin{array}{c}
\bar{z}_{1} \\
g_{1} \\
\vdots \\
\bar{z}_{k-1} \\
g_{k-1}
\end{array}\right]=\left[\frac{b_{k-1}}{\bar{b}_{k-1}}\right],
$$

where $T_{k-1}$ is orthogonal, and $S_{k-1}$ is nonsingular upper triangular with the same number of rows $m$ as $b_{k-1}$. Then at epoch $k$ after obtaining $V_{k}, \bar{z}_{k}$ and $g_{k}$, we perform the following orthogonal transformations:

$$
\tilde{T}_{k}^{T}\left[\begin{array}{c}
S_{k-1} \\
-c V_{k} \bar{P} \\
s I
\end{array}\right]=\left[\frac{S_{k}}{0}\right], \quad \tilde{T}_{k}^{T}\left[\begin{array}{c}
b_{k-1} \\
\bar{z}_{k} \\
g_{k}
\end{array}\right]=\left[\begin{array}{c}
\frac{b_{k}}{\hat{b}_{k}}
\end{array}\right], \quad \bar{b}_{k} \equiv\left[\begin{array}{c}
\bar{b}_{k-1} \\
\hat{b}_{k}
\end{array}\right],
$$

where $\tilde{T}_{k}$ is orthogonal, $S_{k}$ is nonsingular upper triangular, and $S_{k}$ and $b_{k}$ each have $m$ rows. The orthogonal transformations can be implemented to take advantage of the upper triangular structure of $S_{k-1}$ and the diagonal structure of $s I$. But the matrices $\tilde{T}_{k}$ and $T_{k-1}$ are neither formed nor stored. By using similar notation for the transformed noise vector, we get the transformed form of (4.8):

$$
\left[\begin{array}{c}
b_{k} \\
\bar{b}_{k}
\end{array}\right]=\left[\begin{array}{c}
S_{k} \\
0
\end{array}\right] a+\left[\begin{array}{c}
w_{k} \\
\bar{w}_{k}
\end{array}\right], \quad\left[\begin{array}{c}
w_{k} \\
\bar{w}_{k}
\end{array}\right] \sim \mathcal{N}\left(0, \sigma_{\phi}^{2} I_{2 k(m-2)}\right)
$$


Thus by solving the upper triangular system

$$
S_{k} a_{k \mid k}=b_{k}
$$

we obtain $a_{k \mid k}$, the LS estimate of $a$ at epoch $k$. After this we can solve (4.9) to obtain any $x_{j \mid k}$, the estimate of $x_{j}$ at epoch $k$.

Handling the nonlinearity problem $\left(E_{k}=E\left(x_{k}\right)\right)$ is straightforward, see [3].

\section{Real data tests}

We give real data test results here. The data was provided by VIASAT GeoTechnology Inc, a company in Montreal, Canada. The receivers were made by Canadian Marconi Company. The user who held a receiver was walking in an open sky environment. The baseline was about $3 \mathrm{~km}$. The time interval between two consecutive epochs was 1 second. We used the position estimates obtained by VIASAT Geo-Technology software as the "true" positions. Their software used a complex positioning algorithm, and it is believed that the errors in its estimates were about a few centimetres, so the "Error" in Figure 5.1 is suspect by at least this amount. It is also affected by a "bias" in the data, see Section 6 . For comparison, we computed the position estimates not only by the algorithm presented in Section 4 (we took $\sigma=0.001$ in the computation), but also by the algorithm based only on carrier phase measurements presented in [4] and an algorithm based only on code measurements as well. The algorithm based only on code measurements is straightforward. In fact, from the code measurement equation (3.7), we compute the estimate of $x_{k}$ (and $\beta_{k}$ ) by a simple LS method. The errors of the position estimates by the three algorithms are shown in Figure 5.1. There were 6 visible satellites and there were no rising or setting satellites during this testing period (this determined the testing period).

Figure 5.1 shows that using both code and carrier phase measurements provided more accurate position estimates than using code or carrier phase measurements alone. At the beginning the carrier phase based position estimates were less accurate than the code based ones. But later the former became more accurate, and finally became almost as accurate as the estimates from the combined measurements. Including the code measurements accelerated the convergence.

\section{Summary and remarks}

We have presented a recursive LS approach for combining the code and carrier phase measurements for GPS positioning. Our algorithm is numerically reliable since we use numerically stable orthogonal transformations. It is also efficient, since it takes full advantage of the structure of the problem. In [3] this approach is extended to compute estimates of error covariance matrices, and to handle rising and setting satellites.

In some receivers, including those that gave the data we used, there is a hardware delay that is slightly different for the code and carrier phase measurements, and this is sometimes referred to as a hardware bias. In our model we ignored this possible difference, and this can lead to a small error in our estimates. But 


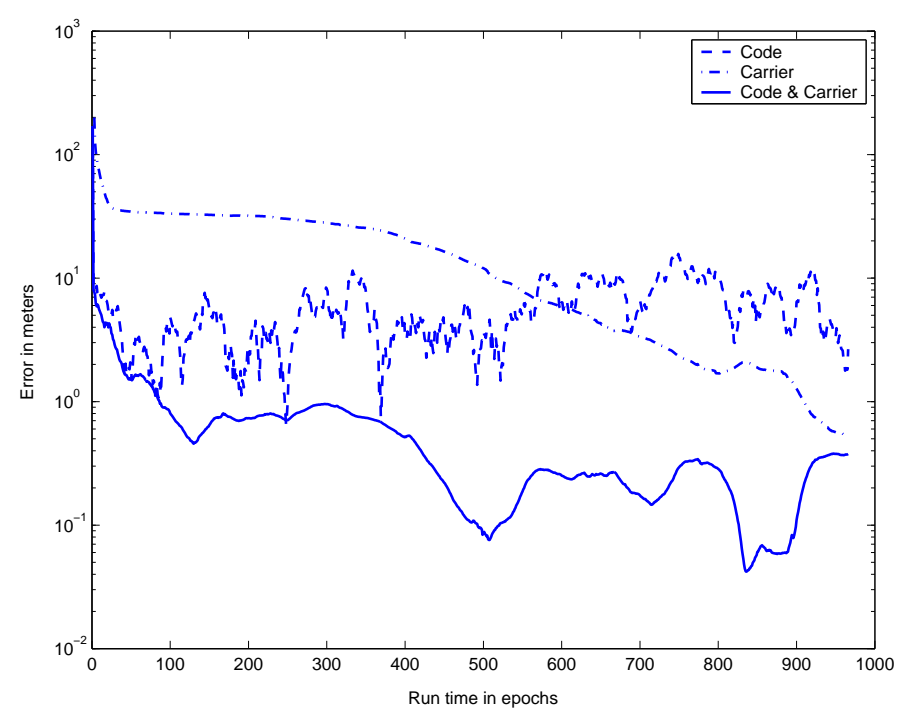

Figure 5.1: Position estimate errors versus time

this is the model which is described in most of the literature, see for example [11, $\S 4.1]$, and even for those receivers with such a hardware bias, the bias appears to be small enough so that this model still leads to sub-metre accuracy for a fixed number of visible satellites, see for example Figure 5.1. But to obtain higher accuracy, or to be able to handle rising and setting satellites accurately when there is such a bias, it might be necessary to include either a single constant delay parameter (since the delay difference tends to be fairly constant under stable conditions), or a time varying delay. However this would lead to a more complicated algorithm. See Tiberius [13] for a treatment of this problem.

Even though we have ignored the above bias as well as some other possible refinements to the model, the computed results on the practical test data (which was subject to such a bias, but restricted to a fixed number of visible satellites) were very positive for such a simple and efficient algorithm.

\section{Acknowledgments}

We would like to thank Liang Qiu and Lan Yin for carrying out the computations, Claude St-Pierre for providing the real data, and Elizabeth Cannon, Richard Langley, Rock Santerre, Christian Tiberius and Jianjun Zhu for helpful discussions. A referee helped us improve the presentation.

\section{REFERENCES}

1. J. Ashjaee, Ashtech XII GPS technology, Proceedings of IEEE PLANTS'90 Position Location and Navigation Symposium, Las Vegas, IEEE, New York, pp. 184-190. 
2. J. D. Bossler, Clyde C. Goad, Peter L. Bender, Using the Global Positioning System (GPS) for geodetic positioning, Bulletin Géodésique, Vol. 54, 1980, pp. 553-563.

3. X.-W. Chang and C.C. Paige, Combined code and carrier phase based GPS positioning with rising and setting satellites, School of Computer Science, McGill University, Montreal, Canada, October 2002, Technical Report SOCS-02.9, 16 pages, http://www.cs.mcgill.ca/resrchpages/tech2002.html.

4. X.-W. Chang and C.C. Paige, An orthogonal transformation algorithm for GPS positioning, to appear in SIAM J. Sci. Comp., 23 pages.

5. C.C. Goad, Optimal filtering of pseudoranges and phases from single-frequency GPS receivers, Navigation, Journal of The Institute of Navigation, Vol. 37, Spring 1990, pp. 249-262.

6. C.C. Goad, B.W. Remondi, Initial relative positioning results using the Global Positioning System, Bulletin Géodésique, Vol. 58, 1984, pp. 193-210.

7. R. Hatch, The synergism of GPS code and carrier measurements, Proceedings of the Third International Symposium on Satellite Doppler Positioning, New Mexico State University, New Mexico, February 8-12, 1982, Vol. 2, pp. 1213-1231.

8. P.Y.C. Hwang and R.G. Brown, GPS navigation; combining pseudo-range with continuous carrier-phase using a Kalman filter, Navigation, Journal of The Institute of Navigation, Vol. 37, pp. 181-196, 1990.

9. X.X. Jin, Algorithm for carrier-adjusted DGPS positioning and some numerical results, Journal of Geodesy, Vol. 71, pp. 411-422, 1997.

10. A. Kleusberg, Kinematic relative positions using GPS code and carrier beat phase observations, Manuscripta Geodaetica, Vol. 10, 1986, pp. 257-274.

11. P. Misra and P. Enge, Global Positioning System, Signals, Measurements, and Performance, Ganga-Jamuna Press, Lincoln, Massachusetts, 2001.

12. P.J. Teunissen, The GPS phase-adjusted pseudo-range, Proceedings of the Second International Workshop on High Precision Navigation, K. Linkwitz and U. Hangleiter (eds), Dümmler, Bonn, 1991, pp. 115-125.

13. C.C.J.M. Tiberius, Recursive data processing for kinematic GPS surveying, $\mathrm{PhD}$ Thesis, Mathematical Geodesy and Positioning, Delft University of Technology, 1998. 